\title{
Barreras para la construcción de la educación inicial como un espacio educativo en comunas rurales
}

\author{
Javier Pineda \\ Rimisp-Centro Latinoamericano para el Desarrollo Rural, Santiago, Chile. \\ Email: jpineda@rimisp.org
}

\begin{abstract}
Resumen: El siguiente artículo presenta los resultados de una investigación que indagó en las barreras que enfrenta la educación inicial por la baja calidad de los centros pre-escolares, la desvalorización de las familias con respecto a la educación infantil, los problemas por la falta de capacidad de la gestión local, y la ruralidad de las comunas en su construcción como un espacio educativo. La metodología fue cuantitativa y cualitativa, y se investigaron centros pre-escolares y actores del municipio que estuvieran relacionados con la educación inicial, en comunas rurales de la región de Coquimbo y el Maule. Los resultados evidencian la importancia de la participación de las familias para la calidad de los centros pre-escolares, y también que los municipios son obstaculizadores para el ejercicio de liderar los centros hacia el éxito. A través de este análisis se propone pensar en una sinergia que parece estar poco abordada en las políticas públicas: esta es, la relación entre primera infancia y educación.
\end{abstract} rurales.

Palabras clave: educación inicial, calidad, familias, gestión local, comunas

\section{Barriers to the construction of early childhood education as an educational space in rural communes}

\begin{abstract}
The following article presents the results of a study that investigated the barriers facing early childhood education because of the poor quality of pre-school centers, the family's impairment to early childhood education, the problems caused by the lack of local management capacity, and the characteristics of rural communes in their construction as an educational space. The methodology of this research was quantitative and qualitative, and pre-schools and municipal actors related to early childhood education in rural communes of the region of Coquimbo and Maule, were investigated. The results showed the importance of family participation in achieving quality on pre schools, and also that municipalities are obstructionist in the exercise of leading preschools to success. Through this analysis we propose to consider a synergy that seems to be not approached enough in public policy: that is, the relationship between early childhood and education rural communes.

Key words: early childhood education, quality, families, local management,
\end{abstract}

\section{Barreiras para a construção da educação inicial como um espaço educativo em municípios rurais}

Resumo: Este artigo apresenta os resultados de uma pesquisa que investigou 
as barreiras que enfrentam a educação inicial; a baixa qualidade da pré-escola; a desvalorização das famílias em relação à educação da primeira infância; os problemas de falta de capacidade de governança local; e a ruralidade das comunas; na sua construção como um espaço educativo. A metodologia quantitativa e qualitativa, onde foram investigados pré-escolas e atores do município, que estavam relacionados com a educação infantil em distritos rurais da região de Coquimbo e Maule. Os resultados mostram a importância da participação da família para a qualidade dos centros pré-escolares e que os municípios estão a dificultar o exercício de encaminhamentos destes centros para o sucesso. Através desta análise propomos considerar uma sinergia que parece ser pouco abordada nas políticas públicas, ou seja, a relação entre a primeira infância e a educação. nas rurais.

Palavras-chave: educação inicial, qualidade, famílias, gestão local, comu-

$$
* * *
$$

\section{El problema}

La educación inicial es la etapa educativa no obligatoria que se brinda a niños menores de seis años y se considera como el ciclo previo a la educación primaria. La evidencia científica ha demostrado que la educación temprana favorece tanto al desarrollo cognitivo, emocional, social de los infantes (Yeide y Kobrin, 2009; Chang y Romero, 2008; Carneiro y Heckman, 2003) como a la economía de un país (Heckman, 2006).

Algunos estudios muestran que la inversión en la primera infancia presenta tasas de retorno positiva, que es mayor que el retorno que genera la inversión en programas sociales y programas de educación a edades mayores (Heckman, 2006). La educación inicial es especialmente beneficiosa para los niños dado que mejora el rendimiento, ayuda a desarrollar habilidades sociales y aumenta las posibilidades tanto de alcanzar mayores logros educativos en las siguientes etapas de la vida escolar, como de lograr una inserción laboral exitosa a largo plazo (Chang y Romero, 2008).

La asistencia a los centros pre-escolares con regularidad es especialmente importante para los niños de familias más pobres, ya que estos hogares tienen menos posibilidades para ayudar a sus hijos a recuperar los tiempos perdidos en el aula (2008).

Sin embargo, en Chile existe un problema de cobertura en la educación inicial, pues según la Encuesta de Caracterización Socioeconómica Nacional (CASEN) la asistencia a los centros pre-escolares es de sólo 52\%. El principal motivo de inasistencia es "por razones personales" ${ }^{1}$, siendo un 95\% el porcentaje de familias que declaran esa causa, más que razones de acceso al establecimiento (4\%) o económicas (1\%). De esto se infiere que los factores que influyen en la asistencia son principalmente familiares, los cuales serán abordados en este artículo. 


\section{¿Existe una sinergia entre políticas de primera infancia y educación?}

Este panorama nos evidencia el problema de la asistencia a educación pre-escolar, más aún que no ha existido una política pública efectiva para mejorarla. El programa Chile Crece Contigo ha sido la política que ha intervenido en la primera infancia ${ }^{2}$, sin embargo, sus prestaciones están enfocadas en los ámbitos de salud, en la atención pre-natal de las madres o en talleres de cuidado y crianza de los niños. (Silva y Molina, 2010). En efecto, el foco del programa es netamente biopsicosocial, es decir, incluye simultáneamente aspectos biológicos, físicos, psíquicos y sociales del niño, donde comienzan a ser asistidos desde que la madre ingresa al sistema de salud en el periodo pre-natal, donde luego se inicia su seguimiento en las etapas de cuidado del niño (post-natal) hasta que cumpla 4 o 5 años de edad. ${ }^{3}$

El programa no tiene un foco claro en promover la importancia de la educación en primera infancia a través de los centros pre-escolares como espacios para la estimulación de los niños, ni menos en apoyar las gestiones locales para mejorar la asistencia a la educación inicial.

El programa Chile Crece Contigo, en lo que respecta a educación, tuvo sólo un objetivo que fue la creación de infraestructura de jardines infantiles y salas cunas: entre el 2006 y el 2010 la oferta pública de salas cunas aumentó de 14.402 a 85.000 y en jardines infantiles de 82.400 a 127.472 (Silva y Molina, 2010).

Más aún, los anuncios de gobiernos en Chile en los últimos años han puesto a la educación inicial como un espacio para mejorar la incorporación de las mujeres al trabajo. En efecto, la mayoría de los estudios aluden sobre la importancia de aumentar el acceso a jardines infantiles y salas cunas para apoyar la feminización del mercado laboral (Encina, 2008).

En síntesis, el programa carece de un componente educativo que implique ir más allá del acceso: los esfuerzos del programa en educación estuvieron puestos en aumentar la cobertura de los jardines infantiles y salas cunas y apoyar la incorporación de la mujer al trabajo (Silva y Molina, 2010).

\section{¿Cómo abordar el problema de la educación inicial?}

El estudio que se aborda en este artículo presenta un análisis de los problema que acarrea la educación inicial, los que se manifiestan en los siguientes ámbitos: 1) La baja calidad de los centros pre-escolares que se expresan en la falta de docentes calificados, infraestructura defectuosa, recursos limitados (Bedregal, 2006) y la frágil relación entre educadoras y familias (Villarroel, 2004; Villarroel y Sánchez, 2002) 2) la baja valorización de 
las familias a los centros de educación pre-escolares (Pineda, 2013), 3) la escasez de capacidades tanto administrativas como técnico-pedagógicas de los gobiernos municipales para enfrentar los problemas de la educación (Muñoz y Muñoz, 2013; Marcel y Raczynski, 2010) y 4) las características de las comunas con alta ruralidad que dificultan el acceso a la educación tanto a las familias como a los profesionales (Pineda, 2013; Williamson, 2004).

Este escenario nos invita a pensar la dinámica en la cual se desenvuelve la educación en primera infancia, bajo el supuesto de que las tensiones que provoca la calidad de los centros pre-escolares, las valorización de las familia a la educación, la gestión municipal y la ruralidad de las comunas son un obstáculo para pensar la educación inicial como un espacio educativo ${ }^{4}$.

El artículo se encuentra organizado de la siguiente manera: a continuación se presenta la metodología del estudio que consistió en estudiar centros pre-escolares en comunas rurales que fueran exitosos y no exitosos en la asistencia de los niños, luego la discusión sobre el efecto que tiene la participación de las familias sobre la calidad de los centros pre-escolares, posteriormente las tensiones entre la crianza de las familias con los centros, a continuación acerca del problema de la gestión municipal para administrar y movilizar el éxito de los centros, y finalmente cómo las condiciones de las comunas rurales afecta a la educación inicial para que se consolide como espacio educativo.

\section{Metodología}

El estudio es de carácter cuantitativo y cualitativo. En primer lugar se llevó a cabo un análisis descriptivo para seleccionar tanto las comunas rurales como centros pre-escolares y, en segundo lugar, se efectuaron entrevistas semi-estructuradas y grupos focales a los actores involucrados con dichos centros. fueron:

Los criterios metodológicos para seleccionar los casos del estudio

- Criterios teóricos:

1) Comunas con alta ruralidad: La hipótesis del estudio fue que en comunas rurales el problema de la asistencia a educación inicial era más crítico debido a: a) mayores tasas de pobreza, b) problemas de conectividad para acceder a servicios educativos, c) déficit en los recursos humanos en los centros educativos y d) falta de capacidad administrativa y técnica-pedagógica de los gobiernos locales para enfrentar los problemas de baja asistencia de los niños.

2) Centros pre-escolares de jardines infantiles y salas cuna de la Junta Nacional de Jardines Infantiles (JUNJI) y la Fundación Nacio- 
nal para el Desarrollo Integral del Menor (INTEGRA): Ambas dependencias administrativas son las principales instituciones de educación inicial en Chile donde asisten niños de entre 0 a 6 años ${ }^{5}$.

3) Tipos de centros pre-escolares: En el caso de los centros preescolares se han investigado los JUNJI Vía de Transferencia de Fondos (VTF), ya que en su mayoría pertenecen a la administración de los municipios y en este estudio nos interesaba conocer la gestión local a estos centros pre-escolares. En el caso de la fundación INTEGRA sólo existe una modalidad que no depende del municipio sino que de la misma fundación, sin embargo, nos interesaba comparar las experiencias entre los centros pre-escolares ${ }^{6}$.

- Criterios cuantitativos:

4) Selección de comunas y centros pre-escolares: Por medio de los datos de CASEN ${ }^{7}$ y de las dependencias JUNJI e INTEGRA se han seleccionado las comunas exitosas y no exitosas del estudio. En primer lugar, se definió como exitosos que no tuviera problemas de asistencia de niños ni falta de oferta o cupos en los centros preescolares y, segundo, no exitosos lo contrario. Pertenecen al primer grupo las comunas de Los Vilos y Longaví, en tanto que al segundo corresponden las de Combarbalá y Parral. Adicionalmente, se investigó un centro pre-escolar de la institución JUNJI VTF en la comuna de Romeral a modo de pre-test para probar los instrumentos de recolección de información cualitativos. Del mismo modo, para el caso de los centros pre-escolares se han seleccionado aquellos que tengan ambos niveles de sala cuna y jardín infantil por cada comuna. El criterio de selección fue el mismo que el de las comunas, es decir, se observó los niveles de asistencia y oferta individualizadamente en cada centro ${ }^{8}$.

\section{- Criterios cualitativos}

5) Instrumentos de recolección de información: Se construyeron pautas de entrevistas semi-estructuradas para efectuarse tanto a actores individuales como para grupos focales, de esta manera se pretendía conocer las experiencias en profundidad de los centros pre-escolares y otros actores involucrados con la problemática.

6) Los actores entrevistados fueron los siguientes:

? Actores relacionados con los centros pre-escolares: El objetivo era conocer las experiencias directas con el tema de la asistencia de los niños a los centros, la participación de las familias, los problemas de la calidad y la relación con los municipios. Los actores entrevistados fueron:

- Educadoras y técnicas de educación parvularia de los centros preescolares: Se realizaron grupos focales.

- Directoras de los centros pre-escolares: Se realizaron entrevistas semi- estructuradas.

- Familias (Apoderados): Se realizaron grupos focales. 
pos focales.

- Familias que no asisten a centros pre-escolares: Se realizaron gru-

- Actores del municipio: El objetivo era conocer la relación entre los actores del municipio con la educación inicial ya sea en los apoyos logísticos, administrativos, pedagógicos y la implementación de políticas como el programa Chile Crece Contigo.

- Director Departamento de Administración de Educación Municipal (DAEM): Se realizaron entrevistas semi-estructuradas.

- Coordinadora Educación Parvularia del Departamento de Administración de Educación Municipal (DAEM): Se realizaron entrevistas semi- estructuradas.

- Encargada Comunal Chile Crece Contigo

- Jefe Departamento de Desarrollo Comunitario (DIDECO): Se realizaron entrevistas semi estructuradas.

7) Se realizaron en total 35 entrevistas semi-estructuradas y grupos focales a los distintos actores.

\section{La díada familia y centro pre-escolar para pensar la calidad de la educación inicial}

Una gran parte de la literatura define educación inicial, infantil, preescolar o parvularia como una etapa para facilitar un buen comienzo en la educación primaria (Leseman, 2009; Heckman, 2006 OECD, 2001). Los centros pre-escolares son espacios para proporcionar el desarrollo de los niños en una amplia gama de destrezas y competencias, que les ayuden tanto a aprender como a aprovechar todas las oportunidades educativas que ofrecen las etapas obligatorias primaria y secundaria (Leseman, 2009). Más aún, asistir a educación inicial en los países con alta desigualdad social y económica adquiere especial relevancia, ya que ayuda a disminuir las desventajas iniciales provenientes de las familias más vulnerables (Castro, Cosso y Valiente, 2012; Andrada y Aguilera, 2011).

A pesar de la evidencia sobre los beneficios de la educación inicial, en Chile la calidad de la misma no es un tema importante en las agendas de gobierno (Castro, Cosso y Valiente, 2012). A la hora de definir calidad de los centros pre-escolares, se tiende a asociar a aspectos de infraestructura, calidad del profesorado, curriculum y a una coordinación eficiente con otros servicios como salud (Bedregal, 2006). No obstante, la coordinación entre los centros pre-escolares y las familias también es fundamental para lograr una mejor calidad (Pineda, 2013). Algunos estudios sobre educación escolar básica ${ }^{9}$ muestran que para las familias de comunidades rurales (principalmente de orígenes indígenas) es de suma importancia que sus hijos asistan a la escuela, pues prevalece un discurso de que el aprendizaje es para “ser alguien en la vida” o "ser mejores” (Villarroel, 2004; Villarroel y Sánchez, 2002). 
Sin embargo, no hay coincidencias entre los discursos entre las familias y educadoras, ya que estas últimas aluden que las familias le dan poca importancia a la educación, ya sea por falta de apego, analfabetismo y/ o razones laborales (Villarroel y Sánchez, 2002). Del mismo modo, en los hallazgos de este estudio se observó que tanto las educadoras como las técnicas en educación parvularia deben enfrentar los desafíos por motivar a familias desinteresadas con la enseñanza de sus hijos a una edad temprana, su asistencia a los talleres y reuniones de apoderados casi siempre es muy baja, lo cual es interpretado por los centros como una falta de motivación y desinformación de las familias sobre las actividades que ahí se realizan: para las familias los centros pre-escolares son vistos como un espacio para el cuidado de los niños, al cual deben recurrir por una necesidad.

"Los papás aún siguen pensando que los jardines son guardería, los mandan muchas veces por necesidad, por trabajar, no pueden dejarlos en la casa (a sus hijos) ¿quién se los va a cuidar?” (Entrevista educadoras y técnicas en parvularia)

A pesar de esta creencia de las familias, el curriculum educativo de los centros pre-escolares no sólo se basa en el cuidado físico de los niños sino que también de actividades pedagógicas que se materializa en una "rutina". Las actividades pedagógicas que se realizan van desde materias como el lenguaje, historias, ciencias naturales hasta matemática, además se trabaja la socialización y la autonomía de los niños.

La baja participación de las familias se ve condicionada por esta falta de información sobre las funciones que cumplen los centros pre-escolares, pues las necesidades que priman para asistir a un jardín infantil o sala cuna son por el trabajo de la madre, apoyo adicional al cuidado, enfermedad o de alimentación.

A pesar que el vehículo de entrada a un centro pre-escolar sea la necesidad, las familias tienden a valorar a largo plazo los beneficios de la educación inicial en sus hijos, sobre todo en los ámbitos de socialización y autonomía. En efecto, los estudios de Villarroel y Sánchez (2002) sobre educación escolar básica en comunidades rurales muestran que las familias valoran que sus hijos aprendan a modales, hábitos, valores y a compartir con otros. A su vez, las familias entrevistadas en este estudio aludían que para ellas era muy importante que sus hijos aprendieran esto a una edad temprana ya que así les beneficiaría a su adaptación en la etapa escolar básica.

Sin embargo, este discurso es más habitual en familias que se han comprometido con los centros pre-escolares, lo cual también es complejo de hacer perdurar dado que depende, en cierta medida, del liderazgo que ejerzan las directoras de los centros pre-escolares por mantener el compromiso de estas familias.

El liderazgo de las directoras es un elemento clave para mantener una efectiva relación centro pre-escolar y familia. Por un lado, son claves para la 
coordinación interna, ya sea en mantener un clima laboral estable entre educadoras y técnicas y, por otro, para lidiar con los conflictos con las familias.

Las familias son exigentes con respecto al buen cuidado que les entregan los centros a sus hijos, esto es: la higiene del niño, alimentación, comportamiento socialmente adecuado (que no haya comportamientos agresivos), entre otros. Las familias no tienen como requisito para elegir un centro pre-escolar la calidad del curriculum educativo, es decir, la rutina que allí se realiza, sino que más bien la calidad de las educadoras y técnicas, lo que para ellas significa que "sepan cuidar bien a sus hijos”. En efecto, la mayoría de los testimonios de familias aluden que para asistir a un centro es más importante la relación de confianza que se establece entre hijo y centro pre-escolar.

“A mí por ejemplo, mi hija aprendió a ir al baño aquí en el jardín, las tías le enseñaron, esas cosas tan chiquitas, que para una como mamá son súper importantes, en eso va la enseñanza y la confianza que uno deposita en las tías y en el jardín” (Entrevista a familias).

“Llegar acá (al jardín) es como llegar a nuestro hogar (...) aquí entre la directora, las tía y hasta la señora del aseo es como una familia, no se hacen diferencias, eso es bueno porque te dan la confianza que ese ejemplo le están dando a tus hijos” (Entrevista a familias).

En general, en los centros pre-escolares existen tensiones entre los equipos de educadoras y técnicas por los problemas que implican las jornadas laborales, la falta de personal y las bajas remuneraciones. Las directoras asumen que estos problemas generan mala disposición para que las educadoras y técnicos en parvularia desempeñen un buen trabajo con las familias. Para las familias es fundamental que se les asegure un buen trato a sus hijos y, por lo mismo, al existir conflictos en los centros pre-escolares se produce una tendencia de deserción a mediano plazo.

En cierta medida, los argumentos anteriores establecen que la calidad de los centros pre-escolares depende en gran parte del profesorado. Las familias exigen que ellas entreguen un buen cuidado, pero ¿qué exigen los centros a las familias? ¿Qué significa que las familias estén desinteresadas sobre las actividades educativas que se realizan en los centros? Si el proceso de construir calidad está mediado por la relación centro pre-escolar y familia, ¿cuál es el aporte de la familia sobre la calidad?

\section{Las tensiones entre las familias y los centros pre-escolares por la crianza de los niños}

La familia es la institución que tiene por prioridad criar a los niños en función de valores, costumbres, prácticas y estilos de vida. La crianza se 
entiende como el proceso de formación y cuidado que se articula entre el dominio de la familia y las instituciones públicas (Rojas, 2010). Por un lado, la crianza se asocia a la vida privada o doméstica, dejando a la familia como su actor principal y, por otro, el Estado es un interventor que resguarda los derechos del niño, incidiendo en su bienestar y desarrollo a través de sus prestaciones tanto en salud como educación (Izzedin y Pachajoa, 2009; Raczynski, 2006).

En primer lugar, la crianza asociada a la familia está determinada por las pautas de crianza (Izzedin y Pachajoa, 2009). Estas se definen en función de las normativas que tienen los padres frente al comportamiento de sus hijos, de modo que ellos estimulan el comportamiento que les parezca adecuado y desincentivan los que no. La familia se encarga de los procesos de socialización de los niños, en los que se aprenden ciertos aspectos de la vida social: se adquiere el lenguaje, las costumbres, los gustos y las preferencias (Valenzuela, et al, 2006).

En segundo lugar, la familia no tiene completa libertad para criar a sus hijos, pues el Estado actúa como un interventor que complementa la crianza de un niño ${ }^{10}$. La crianza se promociona e incentiva por medio de la asistencia a los centros pre-escolares, en los cuales la educación que reciben los niños se enfoca en desarrollar las habilidades correspondientes a cada etapa de desarrollo de la primera infancia (Izzedin y Pachajoa, 2009).

Este escenario nos invita a reflexionar acerca de las tensiones que se producen entre la crianza que reciben los niños en los centros pre-escolares y de las familias. Estas tensiones pueden pensarse en dos niveles: la primera refiere al rol de "voz experta" que cumplen los centros pre-escolares (Bueno y Segura, 2011) y la segunda sobre el significado de "ser buena madre” (Murray, 2012).

La negociación de las pautas de crianza significa para las familias ceder en ciertos aspectos ante la crianza que promueven los centros preescolares. Las familias reconocen que existen pautas que ellos no pueden entregar a sus hijos y que sólo los centros pueden hacerlo: principalmente la socialización y la autonomía.

Sin embargo, la tensión se produce cuando los centros comienzan a demandar exigencias a las familias, como por ejemplo, a hacerse más participes de los procesos de enseñanza de sus hijos. Por un lado, tenemos el problema sobre la falta de interés e información que tienen las familias sobre la educación inicial (que como revisamos en el apartado anterior refiere al problema de asociar un centro pre-escolar como una guardería para el cuidado físico del niño) y, por otro, existen dos desincentivos asociados a una "falta de adaptación" de los niños y a la "falta de estímulos" de los centros pre-escolares para promocionarse.

El primer desincentivo refiere al problema de retención de las familias en los centro pre-escolares dado que sus hijos no han logrado adaptarse a 
las pautas de crianza que ahí se promueven. En general, los centros tienen semanas de adaptación para que los niños logren acostumbrarse a las rutinas que tanto educadoras como técnicos implementan, incluso permiten que las madres estén con los niños los primeros días en el establecimiento para que la transición sea más fácil, aun así, existe altos niveles de fracaso.

“Mi hijo no comía nada (en el jardín) (...) él estaba acostumbrado a más caldos, más sopas, pero igual era mucho sacrificio porque además de vivir lejos cuando lo traía no había caso que él quisiera almorzar” (Entrevista a familia).

El segundo desincentivo se genera por parte de los centros preescolares, producto que no tienen los estímulos para promocionar a las familias la importancia de la educación inicial y acerca de los aprendizajes que facilitan para la crianza. Estos desincentivos se traducen en la falta de prioridad que tienen los municipios en coordinar los centros, ya sea en la administración de las subvenciones, la escasez de capacitación, la falta de iniciativa para solucionar problemas de infraestructura y robos, entre otros aspectos (este punto será desarrollado en mayor profundidad en el siguiente apartado).

Otro nivel de tensión que se genera entre los centros pre-escolares y las familias refiere a las resistencias que encuentran las madres para enviar a sus hijos a los centros porque se ven cuestionadas en su rol de ser "buenas madres". En efecto, los hijos son considerados la prioridad de las madres, el trabajo formal debe quedar en segundo plano (especialmente los primeros años del lactante) y, adicionalmente, la medicina moderna se muestra partidaria de la lactancia materna y al apego seguro. El cuidado de un niño en el hogar legitima a las mujeres como buenas madres (Murray, 2012).

"Ser una buena madre es siempre estar con sus hijos, estar siempre en la casa, eso si ahora hay muchas mamas que trabajan (...) a mí me gustaría mucho trabajar, pero es una cosa por otra, mis hijos pasarían mucho en la calle, no van a ser unos buenos hijos” (Entrevista a familia)

Algunos autores plantean un dilema de decisión política sobre si fomentar la atención a los niños en los hogares o, por el contrario, a promover la asistencia a los centros pre-escolares (Crahay, 2009). Sin embargo, el problema planteado en esta sección fue que ambas son un complemento para el desarrollo integral de los niños ya que se negocian tanto las pautas de crianza de las familias como la de los centros.

\section{El problema de las capacidades de los municipios para enfrentar la educación inicial}

La educación pública se encuentra gestionada y administrada por los municipios de cada comuna, que son continuamente cuestionados por 
sus deficientes capacidades técnicas para enfrentar las demandas de educación (Muñoz y Muñoz, 2013). En efecto, las débiles competencias profesionales para gestionar la educación más la falta de liderazgo técnico-pedagógico son, en parte, los responsables de este problema (Marcel y Raczynski, 2010).

En cuanto a la educación inicial, los hallazgos del estudio nos muestran el siguiente panorama: 1) los municipios tienen una falta de prioridad en conducir el éxito de este nivel educativo y 2) existe una falta de coordinación en los municipios para la aplicación de las políticas de infancia en educación inicial ${ }^{11}$.

Respecto al primer punto, el Departamento de Administración de Educación Municipal (DAEM), a través de la Coordinación Comunal de Educación Parvularia se encarga de administrar la educación inicial en los centros pre-escolares JUNJI Vía de transferencia de fondos (VTF) ${ }^{12}$. Si bien el cargo tiene la función de coordinar temas administrativos y financieros de los centros, también incentivan a los equipos a la búsqueda de logros en diversos ámbitos: estrategias para aumentar tanto las matrículas como la asistencia de los niños y la búsqueda de alternativas para atraer recursos. Sin embargo, los equipos de los centros no legitiman su función de incentivar principalmente por tres razones:

En primer lugar, los salarios y la falta de capacitación. Por un lado, los salarios son un problema que afecta diferenciadamente a los centros JUNJI VTF ya que son los que reciben las remuneraciones más bajas con respecto a otro tipo de dependencia. Por otro lado, los centros JUNJI VTF prácticamente no reciben capacitaciones tanto por parte de los municipios como de la dependencia JUNJI. Los equipos reclaman constantemente este abandono por su perfeccionamiento ya que al compararse con otros tipos de dependencias como JUNJI Clásicos e INTEGRA estas reciben constantes capacitaciones además de existir posibilidades de becas para continuar sus estudios.

En segundo lugar, el manejo de las subvenciones por parte de los municipios es un problema que genera desincentivos para los centros preescolares. La asistencia de los niños a los centros es uno de los temas más controversiales para los municipios ya que su financiamiento depende de ese aspecto. Dado que la asistencia en los centros pre-escolares es baja e inestable, sobre todo en los niveles de sala cuna, los municipios han tenido que buscar métodos para contrarrestar este problema y así lograr la subsistencia de los centros de su comuna. Para ello han creado un "Fondo Solidario”, el cual consiste en que un centro que sea exitoso en la asistencia, tiende a repartir al resto de los centros de la comuna que no son exitosos una parte de su subvención, de manera que puedan solventar los gastos básicos del mismo. En este sentido, el “Fondo Solidario” genera desincentivos tanto para los centros pre-escolares que son exitosos, como los que no lo son, pues por un lado, los exitosos no reciben retribuciones por sus esfuerzos en el trabajo con los niños y por otro, los no exitosos no 
tienen motivación para realizar esfuerzos con la finalidad de mejorar.

En tercer lugar, no existen diagnósticos claros por parte de los municipios, para crear infraestructura de centros pre-escolares. En algunas comunas se levantan más centros de los que la comuna pueda solventar a través de las subvenciones:

"Van a abrir otro jardín VTF más, y nosotras estuvimos conversando con el director DAEM, de que si estamos los cinco (los centros que ya están), dos solventando a los otros tres, ¿va a dar este jardín nuevo que quieren abrir? Y ahora van a haber dos solventando a cuatro, o sea a los seis” (Entrevista directora centro preescolar).

Según las declaraciones de los jefes del DAEM, los diagnósticos se hacen a partir de las demandas de la comunidad y que las bajas asistencia ha sido una problemática que no han sabido afrontar.

Respecto al segundo punto sobre la implementación de las políticas de infancia en educación, parte del problema recae en la falta de coordinación entre el Departamento de Desarrollo Comunitario (DIDECO) -quien es el encargado de ejecutar el Programa Chile Crece Contigo- en relación con el DAEM.

Dentro del programa se articula la Red Comunal Chile Crece Contigo, la cual es coordinada por DIDECO para ofrecer prestaciones a nivel local. Esta red se encuentra compuesta principalmente por los servicios de salud pública, los centros pre-escolares y diversos actores territoriales asociados a la protección de la primera infancia: Carabineros de Chile, Servicio Nacional de Menores (SENAME), entre otros. En la práctica, esta red consiste en reuniones voluntarias mensuales para discutir temas de la contingencia tanto nacional como comunal sobre la primera infancia. Sin embargo, en los casos que se investigaron, la mayoría de los centros pre-escolares dijeron que nunca o rara vez habían sido invitados a las reuniones de la Red, ni siquiera la Encargada de Coordinación Comunal de Educación Parvularia del DAEM.

La mayoría de las directoras de los centros aludían que ser parte de la Red sería una instancia para fortalecer la relación entre la educación inicial y los distintos actores de la primera infancia en su comuna, lo que a su vez serviría para mejorar la calidad de sus servicios. Uno de los desafíos según los actores de DIDECO es articular mejor la Red Chile Crece Contigo, optimizando las relaciones con el DAEM y los otros departamento del municipio involucrados con la primer infancia. Sin embargo, existe una falta de voluntad para llevarse a cabo esta coordinación ya que no hay una intencionalidad para generar dialogo.

“Nosotros (DIDECO) ahora estamos tratando de generar esos espacios, instancias de diálogo. Por lo general las coordinaciones se van 
dando por intervenciones, la misma realidad o problemáticas que van surgiendo que te obligan a coordinarte pero sin la intencionalidad detrás” (Entrevista director DIDECO).

Lo anterior puede deberse a dos razones: Por un lado, la baja preocupación del municipio, dado que las autoridades de turno priorizan políticas y servicios más cercanos a la comunidad en las que la educación queda en un segundo plano (Muñoz y Muñoz, 2013) conlleva a una falta de voluntad por coordinar los distintos agentes de la primera infancia. Por otro lado, la escasez de capacidad técnico-pedagógica de los municipios dificulta la interpretación de las políticas de infancia trayendo como consecuencia un desaprovechamiento de las capacidades de los distintos actores de la primera infancia.

En la gestión local recae la responsabilidad de conducir el proceso de éxito educativo de los establecimientos, ya que tiene como objetivo interpretar e implementar las políticas educacionales. Sin embargo, las políticas educacionales no han fijado las expectativas sobre el aporte que tienen los municipios en los procesos educativos y por lo mismo, sólo se han limitado a temas administrativos y financieros, dejando de lado las gestiones técnica-pedagógicas (Raczynski, 2012). En otras palabras, uno de los problemas que estaría afectando, en cierta medida, la construcción de una capacidad de liderazgo técnica-pedagógica en los municipios, sería la escasa relación que promueve el programa Chile Crece Contigo entre DIDECO y DAEM ya que su falta de coordinación estaría generando una barrera que dificulta fortalecer y mejorar los procesos educativos de la educación inicial.

\section{Los factores asociados a la ruralidad de las comunas que afectan a la educación inicial}

En esta sección se quiere destacar las barreras que generan las características de las comunas rurales para la educación inicial: la dispersión geográfica y la falta de educadoras calificadas.

Las comunas rurales que fueron estudiadas se caracterizaban por poseer jardines y salas cunas en sectores muy dispersos geográficamente. Una gran parte de las familias aludían que su inasistencia a los centros preescolares era por factores de acceso: son muy pocos los centros que tienen transporte para facilitar la asistencia de los niños, pues, en general, deben financiarse de manera privada ya que no tienen apoyo monetario de sus dependencias administrativas.

Algunos municipios han buscado estrategias para solucionar este problema aliándose con establecimientos educacionales vecinos que tengan asociado un transporte escolar para compartirlo con los centros preescolares. Sin embargo, estas iniciativas dependen mucho de la capacidad de liderazgo que tengan los municipios para llevarla a cabo. 
En ciertos sectores la dispersión geográfica entre las familias de zonas rurales es tan pronunciada que ni siquiera existen las posibilidades de acceder periódicamente a los consultorios de las comunas. El problema aparece cuando las familias tienen el deseo de asistir, tanto a las consultas periódicas de los consultorios a través de los servicios que ofrece el programa Chile Crece Contigo como a los centros pre-escolares, y no pueden acceder ya que no existen opciones de conectividad. Más aún, si estas opciones de conectividad no logran ser estables en el tiempo y la familia se encontraba asistiendo es muy probable que deserte.

"Igual era mucho sacrificio porque yo vivo muy lejos, entonces trasladarme para allá... en llegar, lo tenía medio día (a su hijo) estaba como a media hora mi casa del jardín (...) y no había transporte, no existe eso, yo no más en el puro coche... me desviaba por un potrero que hay, entonces era mucho sacrificio, lo tuve por más de un mes en el jardín” (Entrevista a familias).

Otro problema asociado al territorio es la falta de personal para los centros pre-escolares, especialmente profesionales de la educación parvularia. En primer lugar, no existen centros de formación técnica-profesional ni universidades en comunas con alto porcentaje de ruralidad, por lo que existe una escasez de educadoras en los centros pre-escolares ubicados en estas zonas. En segundo lugar, la ruralidad no motiva la migración de profesionales a la comuna para desempeñarse en estos cargos, no sólo por los bajos salarios sino que por las dificultades que significa hacerse cargo de jardines o salas de cunas rurales: baja asistencia, infraestructura defectuosa, problemas conectividad, familias desinteresadas con la educación inicial, entre otros.

El problema anterior ha conllevado que los municipios contraten a profesionales no calificados para desempeñarse en los cargos, es decir, a técnicas graduadas en establecimientos o liceos educacionales (que tienen nivel de técnico en parvularia) para ejercer como educadoras de párvulo. Ante esto, los municipios aluden que cuando se contratan a este tipo de profesionales se agrega en sus contratos un compromiso por parte de ellas donde se estipula que deben continuar con sus estudios profesionales.

Sin embargo, como en dichas comunas no hay oferta de universidades o institutos profesionales y además, estudiar en sectores metropolitanos aledaños significa un costo que muchas educadoras no están dispuestas a tomar, la opción que tienen es matricularse en instituciones que imparten cursos en línea (a distancia).

¿Cuáles son las posibilidades de los centros pre-escolares, ubicados en comunas con alta ruralidad, de ofrecer educación de calidad? ¿Cómo solucionar los factores asociados a la dispersión geográfica? ¿Cómo atraer profesionales a comunas rezagados por su ruralidad? Estas preguntas, asociadas a las comunas rurales, también son barreras que dificultan la cons- 
trucción de la educación inicial como espacio educativo, los cuales deberían ser abordados en las políticas de la primera infancia.

\section{Conclusión}

El principal argumento de este artículo era analizar las barreras que enfrenta la educación inicial para construirse como un espacio educativo, para lo cual se analizaron cuatro dimensiones: la primera consistía en conocer la barrera de la familia que dificultaba la consolidación de la calidad de la educación inicial, pues su desvalorización por este nivel de enseñanza asociado a que los centros pre-escolares sólo facilitan el cuidado físico del niño -, impiden que los centros se formen como un espacio educativo. La familia es uno de los actores claves para lograr una mayor calidad de la educación inicial, pero su bajo involucramiento con esta la ha convertido en una de las principales barreras para generar este potencial.

En segundo lugar, las tensiones que provoca la crianza de un niño también aparecían como una barrera para que las familias se involucraran con los centros pre-escolares. Las pautas de crianza que proponen los centros en contraposición con las impuestas por la familia, generan tensiones que inciden en la deserción de las familias a los centros. En otras palabras, la negociación de las pautas de crianza son determinantes claves para el éxito de los centros pre-escolares por lo que estos deben buscar formas de dialogar (liderazgo) con las familias acerca de los beneficios de las enseñanzas que imparten. Este diálogo que se genera con las familias es un elemento fundamental para incentivarlas a la participación de los procesos de enseñanza de sus hijos en los centros pre-escolares. El "cómo" lo enseñan (confianza) los equipos de educadoras y técnicas es esencial para el efecto que logran sobre la valorización de las familias a la educación inicial.

En tercer lugar, los problemas que genera la gestión local de los municipios promueven desincentivos a los equipos de los centros preescolares. No sólo las bajas remuneraciones o la falta de capacitaciones para el personal sino que también la escaza capacidad administrativa y técnica-pedagógica para manejar las subvenciones y la creación de infraestructura de los centros pre-escolares. En otras palabras, los municipios construyen (y fomentan) un "personal desincentivado" para la educación inicial. Aún queda por saber en futuras investigaciones el efecto que genera para la implementación del curriculum educativo el desincentivo profesional.

En cuarto lugar, las características de las comunas rurales también son una barrera tanto para la formación del profesorado como para el acceso a los servicios de educación inicial. Por un lado, la ruralidad de las comunas no es atrayente para que profesionales migren para trabajar a los centros pre-escolares aislados, ni tampoco existe variedad de oferta de educación superior para que se instruyan en estos sectores. Por otro lado, la falta de posibilidades de acceso para las familias que viven en sectores 
aisladores promueve que la educación inicial sea un beneficio indiferente o provisorio.

La carencia de un foco en educación inicial del programa Chile Crece Contigo se ha traducido en un problema para la articulación de los distintos actores de la primera infancia. La escasa relación entre DIDECO y DAEM genera un desaprovechamiento de las acciones que podrían favorecer a los centros pre-escolares, probablemente no sólo a la formación de la Red Chile Crece Contigo sino que también en articular e implementar de mejor manera las intervenciones de los municipios a la primera infancia.

Finalmente, estas barreras analizadas son un ejemplo de los desafíos que deben afrontar las políticas públicas para mejorar la educación inicial. El reto está en pensar la educación inicial en sí misma, como un espacio reconocido por los beneficios que es capaz de brindar a los niños. Las políticas de infancia deben ir más allá de las metas por una cobertura universal y definir claramente los objetivos de este nivel educativo: ¿facilitar el trabajo femenino? ¿Educar a las familias? ¿Apoyar la crianza? ¿Enseñar a los niños? ¿Quiénes son los agentes educativos de la primera infancia que pueden lograr una correcta coordinación administrativa y técnico-pedagógica? ¿Incluir un foco territorial que considere las diferencias urbano/rurales? Aún queda bastante por hacer, sin embargo el punto de partida para las políticas de infancia debería estar en ser capaces de relacionar la primera infancia con educación. 


\section{Notas}

${ }^{1}$ Según la CASEN las razones personales como motivo de inasistencia son: 1) No es necesario porque lo cuidan en la casa, 2) no me parece necesario que asista a esta edad, 3) desconfío del cuidado que recibirá, 4) se enfermaría mucho, 5) tiene una discapacidad o requiere establecimiento de educación especial.

${ }^{2}$ Los principios rectores del programa son la perspectiva de derechos, la visión integral del desarrollo, la familia como principal agente de la infancia, la importancia del entorno social y comunitario, la protección y apoyo al desarrollo personalizado a través del ciclo de vida y la calidad de las prestaciones como componente fundamental de las garantías. En el año 2009 se promulga la ley 20.379 que crea el Sistema Intersectorial de Protección Social e institucionaliza el Programa Chile Crece Contigo como una política estable (Silva y Molina, 2010).

${ }^{3}$ La coordinación y articulación del programa le corresponde al Ministerio de Desarrollo Social, pero participan otras instituciones que entregan prestaciones especificas a este sistema: Ministerio de Salud (MINSAL), Ministerio de Educación (MINEDUC), Ministerio del Trabajo (MINTRAB), Servicio Nacional de la Mujer (SERNAM), Junta Nacional de Jardines Infantiles (JUNJI), Fundación Nacional para el Desarrollo Integral del Menor (INTEGRA) y Fondo Nacional para la Discapacidad (FONADIS).

${ }^{4}$ Este artículo presenta algunos de los resultados de investigación de un estudio llamado "Experiencias locales para mejorar la asistencia a educación inicial en comunas rurales”, la cual es un producto del programa Cohesión Territorial para el Desarrollo, coordinado por Rimisp-Centro Latinoamericano para el Desarrollo Rural y financiado por el International Development Research Centre (IDRC, Canadá).

${ }^{5}$ En Chile la educación inicial está dividida en seis niveles: 1) Nivel sala cuna menor (84 días a 1 año), 2) nivel sala cuna mayor (1 a 2 años), 3) nivel medio menor (2 a 3 años), 4) nivel medio mayor (3 a 4 años), 5) primer nivel de transición o pre-kínder (4 a 5 años) y 6) segundo nivel de transición o kínder (5 a 6 años). Los niveles 1) y 2) se les denomina en su conjunto "sala cuna" y los 3), 4), 5) y 6) como "jardín infantil", en su conjunto son los “centros pre-escolares".

${ }^{6}$ Según la forma de financiamiento los establecimientos de educación inicial se clasifican en: 1) JUNJI Vía transferencia de fondos (VTF), el cual es parte de la subvención estatal y administrada por los municipios o vía donaciones, 2) JUNJI Clásico que también es estatal pero administrada directamente por esta institución, 3) INTEGRA que funciona vía fiscal y donaciones y 4) Ministerio de Educación (MINEDUC) que tienen tanto niveles medios como de transición, entre ellos existen los con financiamiento municipal y particular subvencionado y 5) privados con financiamiento particular.

${ }^{7}$ La CASEN no es representativa a nivel comunal, por el que el análisis sólo sirvió para generar el marco de referencia de las comunas con mejores y peores niveles de asistencia que se investigarían en la fase cualitativa.

${ }^{8}$ Para el caso de este artículo no es de interés presentar una comparación entre comunas o centros pre-escolares exitosos y no exitosos, sino que analizar las barreras que dificultan la construcción de la educación inicial en su conjunto. Para más detalle 
del estudio puede verse en Pineda (2013).

${ }^{9}$ Es importante destacar que hay una escasez de estudios sobre educación inicial rural, pues la mayoría de las investigaciones existentes están enfocadas en educación básica o bien en los últimos niveles de educación parvularia (niños de 6 años que asisten a kínder). Algunos de estos estudios son: Silva-Peña, Bastidas, Calfuqueo, Díaz y Valenzuela (2013); Williamson, Pérez, Collia, Modesto y Rain (2012); Villarroel (2004); Villarroel y Sánchez (2002).

${ }^{10}$ El 26 de enero de 1990 el Gobierno de Chile se suscribió a la Convención sobre los Derechos del Niño, adoptada por la Asamblea General de la Organización de las Naciones Unidas en 1989, esta instancia está basada en la doctrina de la protección integral del niño, su reconocimiento como sujeto de derecho y el principio del interés superior del niño.

${ }^{11}$ Es importante mencionar que la gestión local que se analiza en este capítulo sólo refiere al caso de los centros pre-escolares JUNJI Vía de transferencia de fondos (VTF), ya que son estos los que les corresponde administrar a los municipios.

${ }^{12}$ No sólo los JUNJI VTF sino que también los que pertenecen a niveles de transición de los establecimientos MINEDUC y especial de lenguaje. 


\section{Bibliografía}

Andrada, Pablo y Aguilera, Wladimir (2011), “El derecho a una educación de calidad” en Cero4 N², Revista Cero4, Editorial Fundación Integra, Santiago.

Bedregal, Paula (2006), "Eficacia y efectividad en la atención de niños entre 0 y 4 años”, en Foco N 79, Expansiva, Santiago.

Bueno, Sebastián y Segura, Nicole (2011), Crianza en la primera infancia: un estudio sobre la configuración de la crianza en familias de la población San Gerónimo de Puente Alto, Tesina de Antropología, Instituto de Antropología, Pontifica Universidad Católica de Chile, Santiago.

Castro, Carlos; Cosso, Jimena y Valiente, José Manuel (2012), Por una mejor educación inicial en Chile: Rol, relevancia y desafíos de la Junta Nacional de Jardines Infantiles [JUNJI]. Serie Sistemas Públicos N7, Centro de Sistemas Públicos, Ingeniería Industrial Universidad de Chile, Santiago.

Carneiro, Pedro y Heckman, James (2003), “Human capital policy” en NBER N 9495, National Bureau of Economic Research, Cambridge.

Crahay, Marcel (2009), “Síntesis y conclusiones” en Educación y Atención a la Primera Infancia en Europa: un medio para reducir las desigualdades sociales y culturales, Agencia Ejecutiva en el Ámbito Educativo, Audiovisual y Cultural, Comisión Europea

Chang, Hedy y Romero, Maria José (2008), Present, engaged, and accounted for: the critical importance of addressing chronic absence in the early grades. NCCP, National Center for Children in Poverty, Mailman School of Public Health at Columbia University, New York.

Encina, Jenny (2008), Chile Crece Contigo: El efecto de la implementación de salas cunas, políticas públicas en participación laboral femenina, pobreza y distribución de ingresos. Programa de las Naciones Unidas para el Desarrollo, Chile.

Heckman, James (2006), "Skill formation and the economics of investing in disadvantaged children” en Science Magazine Vol. 312, n 5782, pp. 19001902.

Izzedin, Romina y Pachajoa, Alejandro (2009), “Pautas, prácticas y creencias acerca de crianza... ayer y hoy” en Liberabit, Revista de psicología, vol. 15, núm. 2, pp. 109-115.

Leseman, Paul (2009), “El impacto de una educación y atención de calidad 
sobre el desarrollo en la primera infancia: estado de la cuestión” en Educación y Atención a la Primera Infancia en Europa: un medio para reducir las desigualdades sociales y culturales, Agencia Ejecutiva en el Ámbito Educativo, Audiovisual y Cultural, Comisión Europea.

Marcel, Mario y Raczynski, Dagmar (2010), La asignatura pendiente. Claves para la revalidación de la educación pública de gestión local en Chile, Uqbar, Santiago.

Muñoz, Cesar y Muñoz, Gonzalo (2013), Desigualdad territorial en el sistema escolar: la urgencia de una reforma estructural a la educación pública en Chile. Documento de trabajo Rimisp Nº 8, Serie Estudios Territoriales, Programa Cohesión Territorial para el Desarrollo, Santiago.

Murray, Marjorie (2012), “ 'Staying with the baby’: intensive mothering and social mobility” en Faircloth, C. Hoffman, D and Layne, L. (editores) Parenting in Global Perspective: Negotiating ideologies of kinship, self and politics, Routledge, London

OECD (2001), Starting strong: Early childhood education and care, en Organization for Economic Co-operation and Development, Paris.

Pineda, Javier (2013), “Experiencias locales para mejorar la asistencia a educación inicial en comunas rurales”, en Documento de Trabajo Rimisp $N^{\circ} 27$ Serie Estudios Territoriales, Programa Cohesión Territorial para el Desarrollo, Santiago.

Raczynski, Dagmar (2012), “Realidad de la educación municipal en Chile: ¿Liderazgo del sostenedor municipal?” en ¿Qué sabemos sobre los directores de escuela en Chile?, Weinstein J., y Muñoz, G. (editores), Fundación Chile y Centro de Estudios de políticas y prácticas en Educación, Santiago.

Ídem (2006), "Política de infancia temprana en Chile: condicionantes del desarrollo de los niños” en Foco N 77, Expansiva, Santiago.

Rojas, Jorge (2010), Historia de la infancia en el chile republicano 18102010, Junta Nacional de Jardines Infantiles, Chile.

Silva, Verónica y Molina, Helia (2010), Cuatro años creciendo juntos: Memoria de la Instalación del Sistema de Protección Integral a la Infancia Chile Crece Contigo 2006-2010, Gobierno de Chile, Santiago

Silva-Peña, Ilich; Bastidas, Karina; Calfuqueo, Luis; Díaz, Juan; y Valenzuela, Jorge (2013) "Sentido de la Escuela para niños y niñas mapuche en una zona rural”, Polis [En línea], 34. Puesto en línea el 22 julio 2013, consultado el 08 marzo 2014.

Valenzuela, Samuel; Tironi, Eugenio y Scully, Timothy (2006), El eslabón perdido: familia, modernización bienestar en Chile, Taurus, Santiago. 
Villarroel, Gladys (2004), “Participación de los hombres rurales en la educación de sus hijos” en Revista Digital eRural, Educación, cultura y desarrollo rural, 1(2). Revisado desde: http://www.revistaerural.cl/GVR.PDF

Villarroel, Gladys, y Sánchez, Ximena (2002), “Relación familia y escuela: un estudio comparativo en la ruralidad” en Estudios Pedagógicos (Valdivia), $\mathrm{N}^{\circ} 28,123-144$.

Williamson, Guillermo (2004), "Estudio sobre la educación para la población rural en Chile”, en F.U.C.R.C. Italiana (Ed.), Educación para la población rural en Brasil, Chile, Colombia, Honduras, México, Paraguay y Perú, pp. 97-170, FAO, Italia.

Williamson, Guillermo; Pérez, Isolde., Collia, Guillermo; Modesto, Francisca y Rain, Nilsa (2012), “Docentes rurales, infancia y adolescentes mapuches”, en Psicoperspectivas, 11(2), pp. 77-96.

Yeide Martha y Kobrin Mel (2009), “Truancy literature review”, en Development Services Group, U.S Department of Justice, Office of Juvenile Justice and Delinquency Prevention, Bethesda.

Recibido:14.02.2014

Aceptado: 08.04.2014 